\title{
(3) The Significance of Abnormalities of Coagulation and Fibrinolysis in Hepatic Disease
}

\author{
-upper gastrointestinal bleeding and hepatic cell necrosis-
}

\author{
Sukeo Yamamoto, M D, Ryonosuke Yoshimura, M D \\ and Teisuke Kamata, M D
}

The Third Department of Internal Medicine, Osaka
City University School of Medicine, Osaka

The haemorrhagic diathesis of fluminant hepatitis or liver cirrhosis has been considered to be due to the hypocoagulability following hepatocellular failure. In liver cirrhosis we have concluded that the mechanism of plasmin activation mainly depends on the increase of tissue activator which releases plasminogen activator into the circulating blood.

Recently much attention has been paid on the participation of disseminated intravascular coagulation (DIC) associated with haemorrhagic diathesis or liver cell necrosis.

Therefore the re-investigation of coagulation and fibrinolysis in hepatic disease is necessary.

On the basis of above evidence, the clinical, laboratory and histopathological findings related to the mechanism of upper gastrointestinal bleeding or hepatic cell necrosis in association with local intravascular coagulation were studied. The results and conclusions of our work are summarized as follows:

1) Liver cirrhosis

The relation between oesophageal bleeding and abnormal coagulation and fibrinolysis was examined.

Oesophageal bleeding was frequently seen in those cases of minimal or negative varices. Blecding occurred as a generalized oozing from the mucous membrane and was sometimes associated with hypocoagulability and increased fibrinolysis. In terminal cases, primary fibrinolysis increased quickly with exercise or stress and was associated with decreased coagulation factors and changes in $\alpha_{2}$-macroglobulin, $\alpha_{1}$-antitrypsin and antithrombim III.

It was very difficult to separate DIC and liver cirrhosis complicated by DIC and we did not find any cases of the latter in our study. ${ }^{125}$ I-fibrinogen turn over rate, serial dilution protamine sulphate test (SDPS), FDP and PIVKA inhibitor index were used to establish the diagnosis.

2) Fulminant hepatitis

The studies on blood coagulation and fibrinolysis were performed in 9 cases with fluminant hepatitis.

All cases showed hypocoagulability due to markedly low levels of coagulable activity. Only one case complicated by DIC was found and heparin was used in treatment. The pathogenesis was also discussed.

3) Experimental endotoxin DIC and liver cell necrosis.

E. Coli endotoxin was in jected into rabbit portal vein and 24 hours later a ten-fold dose of endotoxin was injected in an ear vein. 
Extensive liver necrosis was found which was complicated by fibrin micro thrombi as shown by PTAH staining in the necrotic areas. Similar changes were found in the lungs, kidneys and intestinal tract. In fluminant hepatitis associated with DIC, the pathogenesis and haemorrhagic diathesis were ressembled to that found in our experimental study. 